\title{
O EFEITO DA AMPLITUDE E DA PROFUNDIDADE DA CAPACIDADE DE ABSORÇÃO NO DESEMPENHO EM INOVAÇÃO EM NOVOS EMPREENDIMENTOS INSERIDOS EM ECOSSISTEMAS DE INOVAÇÃO
}

Gloria Charão Ferreira ${ }^{1}$ Jorge Oneide Sausen ${ }^{1}$

${ }^{1}$ Universidade Regional do Noroeste do Estado do Rio Grande do Sul/Programa de Pós-Graduação em Desenvolvimento Regional PPGDR 


\title{
O EFEITO DA AMPLITUDE E DA PROFUNDIDADE DA CAPACIDADE DE ABSORÇÃO NO DESEMPENHO EM INOVAÇÃO EM NOVOS EMPREENDIMENTOS INSERIDOS EM ECOSSISTEMAS DE INOVAÇÃO
}

\begin{abstract}
Resumo: Inovação depende fundamentalmente da criação de conhecimento que possa ser traduzido em elementos com o potencial de aplicação prática, sendo movida pela habilidade em estabelecer relações, detectar oportunidades e beneficiar-se delas. Nesse contexto, os novos empreendimentos enfrentam um grande desafio frente à complexidade presente nas dinâmicas ecossistêmicas, pois se por um lado apresentam uma especializada capacidade tecnológica, por outro, precisam de um conhecimento tecnológico mais amplo que lhes possibilitem criar e compartilhar novos conhecimentos (Velu, 2015; Larrañeta et al., 2016). Sendo assim, a abordagem dessa proposta de pesquisa destaca o papel das características do conhecimento e, ao fazê-lo, preenche uma lacuna importante no campo das capacidades dinâmicas e empreendedorismo. Portanto, esta proposta de investigação tem-se como objetivo "analisar o efeito da amplitude e da profundidade da capacidade de absorção no desempenho em inovação incremental e radical em novos empreendimentos inseridos em ecossistemas de inovação".
\end{abstract}

Palavras chaves: Amplitude da capacidade de absorção. Profundidade da capacidade de absorção. Desempenho em Inovação. Novos empreendimentos.

\section{Introdução}

Em um cenário marcado por mudanças aceleradas, ambientes dinâmicos e turbulentos caracterizados em grande parte pela revolução tecnológica e informacional, o desempenho em inovação acaba por ser uma preocupação crescente nas empresas. Contudo, ao mesmo tempo em que as empresas são confrontadas com o desafio de inovar, também se deparam com suas limitações internas, situação que irá demandar a busca e a incorporação de conhecimento externo, bem como o desenvolvimento de novos modelos organizacionais para ultrapassar tais obstáculos.

Sob essa perspectiva, o crescimento de uma empresa está intimamente alinhado com a aquisição de conhecimento e sua aplicação, fato particularmente preocupante para as empresas que estão em seus estágios iniciais. De fato, estudos argumentam que uma dificuldade encontrada pelos novos empreendimentos reside na falha em reconhecer e buscar fontes externas de conhecimento, situação agravada pela restrição de recursos e pressão para alcançar resultados (Helfat \& Lieberman, 2002; Helfat \& Winter, 2011; Kor \& Mesko, 2013).

Dada a importância da aprendizagem para o desempenho dos novos empreendimentos, considera-se relevante compreender como se relacionam com o ambiente externo no sentido da busca de informações relevantes que lhes permitam sobreviver e se tornarem competitivos. Acrescenta-se a isso o fato de estudos apontarem que a capacidade de absorção (ACAP) apresenta uma natureza cumulativa e dependente do caminho (Cohen \& Levinthal, 1990; Zahra $\&$ George, 2002). Assim, considera-se surpreendente a pouca atenção que tem sido dada ao desenvolvimento da ACAP em novos empreendimentos que tipicamente tem memória

DOI: $10.14211 / x i-$ egepe-118030 
organizacional limitada, mas que precisam aprender rápido a fim de sobreviverem e terem sucesso.

Nesse sentido, as empresas cada vez mais buscam ambientes ricos em possibilidades de cocriar produtos e colocá-los no mercado, fato que suscitou estudos sobre clusters industriais, que preveem uma territorialidade e interdependência em seus conceitos, alianças estratégicas, e redes de negócios (Porter, 1998, 2003; Nambisan \& Baron, 2013; Patel et al., 2016). No entanto, há setores que requerem recursos e conhecimentos que muitas vezes encontram-se dispersos não apenas em uma região geograficamente delimitada em função de uma base de conhecimento complementar e proximidade cognitiva. Desta forma, surgem estudos que abordam os ecossistemas, visto que os modelos anteriores passam a não ser mais suficientes para explicar a complexidade presente na inovação.

Sendo o contexto dos ecossistemas o espaço que se caracteriza pelo esforço colaborativo de um conjunto diversificado de atores para a inovação, tópicos como a colaboração entre organizações, a criação e a captura de valor têm atraído um interesse crescente de profissionais e estudiosos (Adner, 2006; Adner \& Kapoor, 2010).

Não obstante, tem havido apelos para que futuras pesquisas contribuam para a compreeensão dos aspectos que envolvem a dinâmica organizacional dos ecossistemas de inovação (Autio \& Thomas, 2014; Gawer \& Cusumano, 2014), tópicos que provavelmente terão implicações substanciais não apenas para praticantes e acadêmicos, mas também para formuladores de políticas públicas cujos esforços são direcionados para promover o bem-estar econômico dentro de setores, regiões e nações.

Todavia, partindo-se do entendimento que os ecossistemas são sistemas abertos que exercem influência, mas que também são influenciados por seu contexto, compreende-se que o conceito de capacidade de absorção (ACAP) deva ser traduzido para esse cenário, tendo em vista que o conhecimento é um dos vetores importantes para a adoção de estratégias que visem a promoção do seu crescimento e de sua competitividade.

Assim sendo, a importância da ACAP está assente na promoção e sustentação da inovação, na medida em que os fluxos de conhecimento reabastecem a base de conhecimento da empresa, bem como estimulam as atividades associadas à inovação tecnológica (Zahra \& George, 2002; Zahra, 2008; Escribano et al., 2009; Ferreira \& Ferreira, 2017, 2020). Nessa perspectiva, uma forma adequada de avaliar o efeito da ACAP na inovação tecnologica é considerar sua amplitude versus profundidade (Vasudeva \& Anand, 2011; Zahra et al., 2015).

Considera-se a amplitude e a profundidade da ACAP uma nova tipologia nos estudos que abordam esse tema, tendo assim uma perspectiva diferente dos estudos anteriores (Cohen \& Levintahl, 1990; Zahra \& George, 2002; Fosfuri \& Tribó, 2008; Forés \& Camisón, 2015; Ferreira \& Ferreira, 2017, 2020). Esta nova tipologia difere especialmente dos conceitos de capacidade de absorção potencial (PACAP) e capacidade de absorção realizada (RACAP), introduzidos no modelo de Zahra e George (2002), na medida em que essas divisões têm como base a dimensão do processo e, ao serem consideradas a amplitude e a profundidade explora-se um aspecto até então negligenciado na literatura (Lane et al., 2006), ou seja, tem-se como foco as características do conhecimento absorvido.

Portanto, compreende-se que ecossistemas são terrenos férteis para a aprendizagem e trocas, mas que esse processo irá exigir determinadas capacidades sendo uma delas, a capacidade de absorção. Sob essa perspectiva, considera-se crucial compreender de que maneira novos empreendimentos, em sua maioria inexperientes e com poucos recursos, desenvolvem a capacidade de absorver informações externas, e empregam esse esforço em suas 
atividades de inovação. Responder essa questão, para além de um desafio, pode enriquecer nossa compreensão dos fundamentos da vantagem competitiva de novos empreendimentos, bem como contribuir para o aprimoramento e construção de teorias nessa área.

Assim, entende-se que as contribuições desta proposta de estudo estão relacionadas com a oportunidade de que seus resultados possam suprir algumas lacunas apontadas na literatura, nomeadamente:

I. A carência de estudos que abordem a ACAP sob a perspectiva da amplitude e da profundidade do conhecimento;

II. A escassez de estudos que relacionem a amplitude e a profundidade da ACAP de novos empreendimentos pertencentes a um ecossistema de inovação;

III. A falta de estudos que relacionem empiricamente a amplitude e a profundidade da ACAP com as tipologias de inovação, nomeadamente, a incremental e radical;

IV. A carência de abordagens que considerem a ACAP em outros contextos e níveis.

Portanto, na busca de suprir as lacunas mencionadas, tem-se como objetivo "analisar o efeito da amplitude e da profundidade da capacidade de absorção no desempenho em inovação incremental e radical em novos empreendimentos inseridos em ecossistemas de inovação".

No que se refere aos conceitos norteadores deste estudo, quando ACAP tem-se como foco as características do conhecimento absorvido, ou seja, sua amplitude e sua profundidade (Zahra et al., 2015). Desempenho em inovação é aqui percebido pelo grau de novidade, sendo este classificado como (1) inovação radical, e (2) inovação incremental (Freemann, 1988). Do mesmo modo, entende-se ecossistema de inovação como uma síntese ou arranjos colaborativos em que organizações combinam suas ofertas individuais numa solução coerente e voltada a atender os clientes (Adner, 2006). Neste estudo serão considerados novos empreendimentos aqueles com até oito anos de existência (McDougall et al., 1992; Larrañeta et al., 2016).

O artigo está organizado da seguinte forma: após esta Introdução, a Seção 2, apresenta a fundamentação teórica e as proposições de estudo; enquanto a Seção 3 apresenta as considerações finais e implicações.

\section{Fundamentação Teórica e Proposições de Pesquisa}

\subsection{Capacidade de Absorção - ACAP}

Com o trabalho de Cohen e Levinthal, em artigo publicado em 1989 no Economic Journal, a ACAP passa a ser aplicada ao contexto das organizações. Do ponto de vista conceitual, Cohen e Levinthal $(1989,1990)$ compreendem-na como sendo a habilidade da empresa de identificar o valor da informação, assimilar e aplicar para fins comerciais, sendo esta fundamental para a sua capacidade inovadora

Com os estudos de Zahra e George (2002, p. 186), o conceito de ACAP é ampliado considerando-a como "um grupo de rotinas e processos organizacionais pelos quais as empresas adquirem, assimilam, transformam e aplicam o conhecimento para produzir uma capacidade organizacional dinâmica". O modelo apresentado pelos autores, além de propor a distinção de dois componentes, nomeadamente, da capacidade de absorção potencial (PACAP), e da capacidade de absorção realizada (RACAP), também contempla dois antecedentes (fontes de conhecimentos complementares e a experiência dos funcionários), e três fatores moderadores (desencadeadores de ativação, mecanismos de integração social, regime de apropriabilidade). 
Com a evolução dos estudos, Zahra et al. (2015), apresentam uma maneira de conceitualizar o efeito da ACAP na inovação tecnológica considerando sua amplitude (denominada em inglês Breadth of Absorptive Capacity - BACAP) versus sua profundidade (denominada em inglês Depth of Absorptive Capacity - DACAP). A amplitude e a profundidade da ACAP configuram-se como uma nova tipologia, diferindo especialmente dos conceitos de PACAP e RACAP apresentados no modelo de Zahra e George (2002), na medida em que essas divisões têm como base a dimensão do processo e, neste caso, ao serem consideradas sua amplitude e profundidade tem-se como foco as características do conhecimento (Lane et al., 2006).

No estudo realizado por Zahra et al. (2015), os autores discutem e apresentam uma maneira de conceitualizar o efeito da ACAP na inovação tecnológica considerando sua amplitude versus sua profundidade. A amplitude irá analisar se o conhecimento contido na ACAP da empresa é abrangente e, dentro de um continuum, pode ser considerada estreita ou ampla. Assim, uma ampla ACAP pode propiciar à empresa uma vasta gama de opções quando se trata de inovação tecnológica. Em contraste, a profundidade refere-se ao grau em que a empresa desenvolveu um tipo de especialização, ou expertise em um domínio tecnológico específico, podendo ser superficial ou profunda (Zahra et al., 2015).

Assim, para os autores essas dimensões podem fornecer algumas ideias sobre o valor estratégico da ACAP em relação à inovação tecnológica (inovação de produto e inovação de processo), como mostrado na Figura 1.

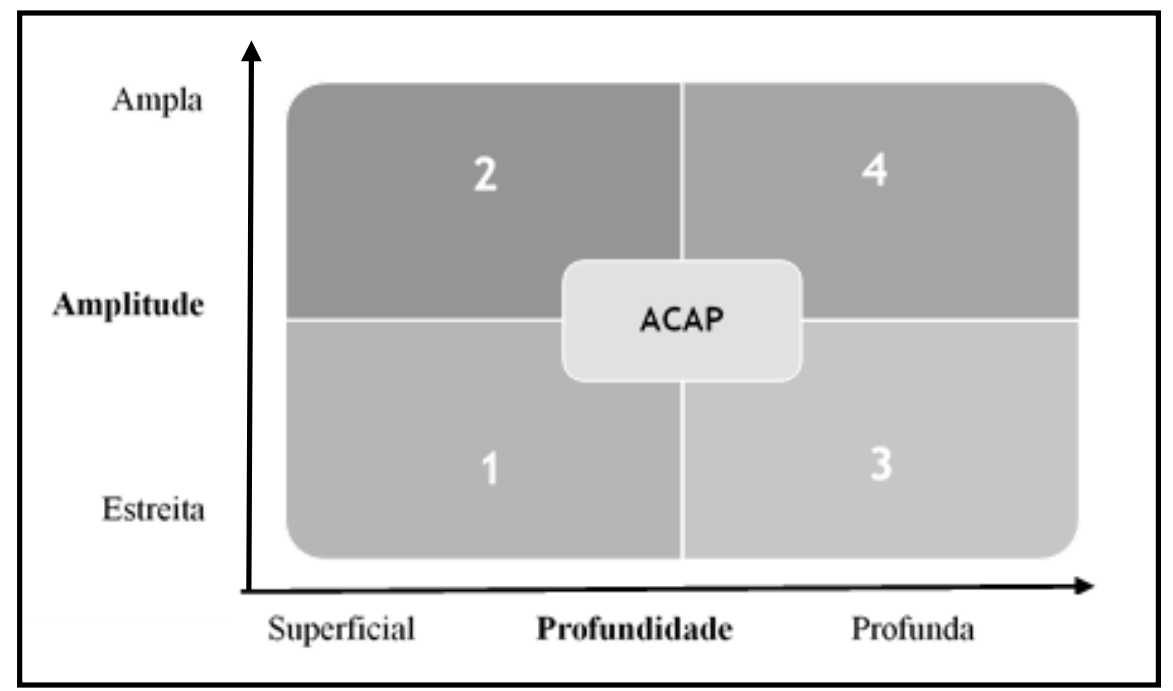

Figura 1 - Profundidade versus Amplitude ACAP e Inovação Tecnológica Fonte: adaptada de Zahra et al. (2015, p. 3)

- No Quadrante 1 - estão as empresas cuja ACAP é superficial e estreita em função de um excesso de especialização, ou a falta de investimentos sustentados em P\&D e outras atividades inovadoras.;

- No Quadrante 2 - descreve a situação de empresas que possuem conhecimento em diferentes áreas, mas não suficientemente especializados. A empresa pode se beneficiar da amplitude de sua ACAP devido à sua facilidade na atualização de seus produtos; 
- No Quadrante 3 - caracteriza as empresas que tem profundo conhecimento em alguns campos, fato que pode ser propício para a construção e proteção de um nicho viável;

- No Quadrante 4 - há a combinação de conhecimento amplo e profundo, oferecendo à empresa oportunidades para criar novas plataformas de produtos ou adicionar mais linhas a produtos existentes por meio de upgrades.

Para os autores, o estudo da amplitude e da profundidade proporciona algumas perspectivas sobre o valor estratégico da ACAP frente à inovação tecnológica, por exemplo, uma empresa cuja ACAP é superficial e estreita em função de um excesso de especialização, terá como resultado uma limitada capacidade de inovar. Embora a entrada de novos conhecimentos auxilie em certa medida a organização, seus benefícios terão dificuldade de se materializar devido à limitada ACAP. Porém, há empresas que apresentam profundo conhecimento em alguns campos, fato que pode ser propício para a construção e proteção de um nicho viável. Entretanto os autores alertam que, ao longo do tempo, essa profundidade pode se tornar uma desvantagem estratégica em ambientes dinâmicos.

O estudo realizado por Farazi et al. (2019), sobre parcerias estratégicas e amplitude e profundidade da base de conhecimento em empresas americanas, refere que pequenas empresas, em função de recursos limitados, concentram-se em domínios específicos (competências essenciais) desenvolvendo assim uma profunda base de conhecimento em poucas áreas críticas. Nesse aspecto, os autores referem esse nível de aprofundamento acaba por facilitar parcerias com empresas que possuem uma ampla base de conhecimento, na medida em que os riscos comportamentais são menores, ou seja, os esforços do dia-a-dia são alocados à aliança e não a outras atividades. No entanto, o conhecimento especializado pode impossibilitar que a empresa responda de forma rápida e flexível às mudanças ocorridas no ambiente ou frente a resultados inesperados, o que acaba por tornar menos atraentes determinadas parcerias.

Do ponto de vista de uma ampla base de conhecimento, a empresa passa a ter uma posição melhor para combinar tecnologias relacionadas de maneira mais complexa e é mais flexível e adaptável para responder a um ambiente em mudança (Srivastava \& Gnyawali, 2011; Farazi et al., 2019). Tal observação corrobora evidências apontadas por outros estudiosos, visto que suas pesquisas apontam que uma base de conhecimento diversificada permite que a empresa responda de maneira mais flexível a vários requisitos e contingências tecnológicas (George et al., 2002; Zhang et al., 2007 Zhang, \& Baden-Fuller, 2010).

\subsection{Desempenho em Inovação}

Do ponto de vista de Tidd e Bessant (2015), a inovação é algo novo que agrega valor, sendo movida pela habilidade da organização em estabelecer relações, detectar oportunidades e beneficiar-se delas. Para esses autores, o processo de inovação é formado por 4 fases e apenas se as empresas conseguirem lidar com todo o processo é que a inovação será bem sucedida: (i) Fase da Busca - onde a empresa analisa o cenário interno e externo com o objetivo de detectar sinais relevantes no ambiente sobre oportunidades para mudança; (ii) Fase da Seleção - tendo como base as definições estratégicas da empresa, decidir quais as variantes com mais possibilidades de auxiliar o seu crescimento e desenvolvimento; (iii) Fase da Implementação onde a empresa transforma as ideias em realidade, empenhando energias e recursos escassos para fazer algo diferenciado; (iv) Fase da Captura de Valor por meio da inovação - fase em que 
a empresa precisa assegurar que os esforços empregados são justificáveis, levando em consideração os benefícios advindos da inovação.

De acordo com o Manual de Oslo (OCDE, 2005) a inovação pode ser classificada quanto ao tipo, podendo ser: (i) inovação de produtos: consideradas mudanças substanciais nas características e/ou composição dos produtos ou serviços; (ii) inovação de processos: mudanças significativas no método de produção ou de distribuição; (iii) inovações de marketing: referemse às mudanças no design do produto, na sua embalagem, no estabelecimento de novos métodos de precificação e na criação de novos mercados; e (iv) inovações organizacionais: estão relacionadas à criação e desenvolvimento de novas formas organizacionais, bem como de mudanças na prática de negócios nos ambientes interno e externo da empresa.

Outra tipologia utilizada em estudos empíricos leva em consideração o grau de novidade, recebendo a denominação de "inovação radical", quando referente ao desenvolvimento e introdução de um novo produto, processo ou forma de organização da produção inteiramente nova. Esse tipo de inovação pode representar uma ruptura estrutural com o padrão tecnológico anterior, originando novas indústrias, setores, mercados, como gerar redução de custos e aumento de qualidade em produtos já existentes; e "inovação incremental", quando referindose à introdução de qualquer tipo de melhoria em um produto, processo ou organização da produção dentro de uma empresa, sem alteração na estrutura industrial (Freemann, 1988).

\subsection{Ecossistema de Inovação}

O termo Business Ecosystem foi cunhado por Moore (1993) em seu artigo da Harvard Business Review "Predators and Prey" e posteriormente conceituado em seu livro "The Death of Competition" (Moore, 1996). Tais redes em torno de uma plataforma de tecnologia não são diferentes dos ecossistemas biológicos. Eles consistem em múltiplos parceiros - ou espécies desempenhando papéis diferentes, eles precisam uns dos outros para o sucesso e sobrevivência, coevoluem e cooperam para criar um ambiente de negócio fértil, bem como competem por sua parcela de recursos.

Desta forma, o conceito de ecossistemas de negócios, e mais especificamente, ecossistemas de inovação, refere-se a uma rede frouxamente interconectada de empresas que coevoluem capacidades em torno de uma inovação, o que acaba por criar uma interdependência entre os atores (Moore, 1993; Zahra \& Nambisan, 2011). Em outras palavras, um ecossistema de inovação compreende um conjunto de sistemas catalisadores, permanentes ou temporários, não geográficos, interdependentes, onde seus atores competem e cooperam com o objetivo de gerar um desenvolvimento tecnológico e inovações que atendam as demandas de mercado.

Segundo Adner e Euchner (2014), um conjunto adicional de fatores e atores precisam se unir para gerar valor a inovação. Esse conjunto maior de atores é o ecossistema de inovação, sendo importante tornar esse conjunto de dependências o mais explícito e cedo possível. Por sua vez, os estudos de Spinosa, Schlemm e Reis (2015), definem ecossistemas de inovação como sendo ativos de competitividade baseados na economia do conhecimento, total ou parcialmente integrados em espaços urbanos, e que são capazes de promover a cooperação regional, bem como o desenvolvimento socioeconômico. Para esses autores, a dinâmica ecossistêmica deve ser pautada no empreendedorismo e na inovação, sendo o último resultado do primeiro, visto que ambos são importantes na busca da competitividade na economia de conhecimento. 
A partir do exposto, percebe-se que a dinâmica presente nos ecossistemas de inovação acaba por fomentar ações colaborativas entre seus atores, sendo uma importante fonte de geração de conhecimento e de transformação do território, visto ser neste onde os atores locais desenvolvem suas atividades de inovação, compartilham conhecimento e estabelecem suas parcerias.

\subsection{A Relação entre Amplitude e Profundidade da Capacidade de Absorção e Desempenho em Inovação em Novos Empreendimentos Inseridos em Ecossistemas de Inovação - Proposições de Pesquisa}

Tem sido pouco questionado que o conhecimento é um recurso fundamental para a inovação. Entretanto, cada vez é mais frequente empresas que ao reconhecerem a importância do conhecimento, buscam acessá-lo através do envolvimento em redes globalmente dispersas, onde novos empreendimentos e empresas de pequeno porte cooperam, competem, e procuram partilhar o conhecimento. Percebe-se que o lócus da competição e da inovação começou a mudar de empresa individual para empresas que juntas buscam desenvolver e adquirir as inovações necessárias para melhor servir os seus clientes. Desta forma, os ecossistemas são considerados um terreno fértil para a criação de novos empreendimentos de diferentes tipos (Zahra \& Nambisan, 2011), que apesar de possuirem características distintas, em muitos momentos são complementares e fundamentais para a sobrevivência do ecossistema.

À luz desses ambientes dinâmicos e mutáveis, tais considerações levam a conceber a inovação não mais dentro de uma lógica linear e sequencial, na medida em que envolve um processo interativo, ou seja, inovar é o resultado da interação entre a empresa e o meio ambiente (Ferreira et al., 2013; Ferreira et al., 2015). Essa interação permite aceder informações que de algum modo possibilitam ao ecossistema um aumento na sua capacidade de gerar inovações (Chesbrough, 2010). Por outro lado, seria imprudente pensar que por conta da existência de um ambiente rico em oportunidades de inovação, qualquer empresa teria capacidade em detectálas e utilizá-las efetivamente.

Tal fato pode ser explicado pela própria dinâmica desse tipo de ambiente, visto que em ecossistemas de inovação o conhecimento incorpora diferentes códigos e características que são muitas vezes difíceis de serem decifrados. Paradoxalmente, o sucesso e a sobrevivência desses ecossistemas dependem em grande parte da capacidade de seus integrantes de criar e compartilhar novos conhecimentos, aproveitando a riqueza das oportunidades existentes em suas redes. Desta forma, o desenvolvimento da ACAP pode configurar-se como um grande desafio quando se leva em consideração a complexidade presente nas dinâmicas ecossistêmicas.

Nesse sentido, Zahra et al. (2015) relatam que a amplitude e a profundidade da ACAP estão intimamente relacionadas ao desempenho em inovação, porém ao longo do tempo ambas podem se tornar uma desvantagem em ambientes dinâmicos. Os autores alertam para o fato de que pode haver limites superiores aos ganhos potenciais de ter uma ACAP bem desenvolvida, ou seja, além um limiar, altos níveis de ACAP pode canalizar o conhecimento externo diverso e novo para modelos mentais existentes, impedindo que as empresas avancem para novos campos tecnológicos. Segundo os autores, para que isso não ocorra a empresa precisa buscar o equilíbrio entre a profundidade e a amplitude de conhecimento que compõe a ACAP, o que irá promover e estimular a aprendizagem organizacional. Portanto, supõe-se que: 
P1: A amplitude da capacidade de absorção de novos empreendimentos, tem um efeito positivo na profundidade da capacidade de absorção.

Sob essa perspectiva, se por um lado a amplitude da ACAP pode permitir que a empresa identifique e adquira conhecimentos diversos e heterogêneos que, ao ampliar a sua base de conhecimento, aumentam as suas oportunidades de inovação, por outro lado, essa diversidade de conhecimento pode se transformar em um passivo para os diferentes atores do ecossistema. Além disso, deve-se considerar que a disponibilidade cognitiva dos tomadores de decisão, bem como da própria estrutura do ecossistema, apresenta limitações no sentido de abarcar uma grande variedade de parceiros (Zahra et al., 2015).

De forma semelhante, os autores referem que o efeito da profundidade da ACAP pode ser considerado crescente quando explicado pelo fato de que interações mais próximas diminuem as barreiras para transferência de conhecimento, especialmente conhecimento tácito. Outra vantagem está no fato de que poderá auxiliar a empresa a identificar e adquirir conhecimento especializado que irá promover alterações incrementais em seus produtos ou processos. A inversão na qual os retornos se tornam decrescentes pode estar associada a um efeito temporal, ou seja, a medida que as empresas se tornam muito próximas a assimetria de conhecimento entre ambas é reduzida e, dessa forma, o aprendizado também, o que poderá afetar o desempenho em inovação. Essas observações sugerem as seguintes proposições:

P2: A amplitude da capacidade de absorção de novos empreendimentos, tem um efeito positivo no seu desempenho em inovação.

P3: A profundidade da capacidade de absorção de novos empreendimentos, tem um efeito positivo no seu desempenho em inovação.

No que se refere ao grau de novidade da inovação, Ritala e Hurmelinna-Laukkanen (2013) consideram que um fator restritivo para o desenvolvimento de inovação radical está assente na falta de uma base de conhecimento diversificada, visto que o conhecimento necessário para uma inovação dessa magnitude muitas vezes encontra-se em áreas nunca antes exploradas pela empresa. Entretanto, uma questão que não pode ser negligenciada reside no fato de que em um ambiente dinâmico, a busca de uma posição competitiva depende da capacidade da empresa expandir suas fronteiras, ampliar e adquirir conhecimentos fora de seu domínio e inovar radicalmente.

Na visão de Tidd e Bessant (2015), apesar de muitas empresas serem capazes de implementar novos projetos, e se beneficiarem de sua ACAP, ainda assim falta-lhes a capacidade para inovações radicais em decorrência da dificuldade que apresentam em perceber onde e como adquirir novos conhecimentos para além de suas fronteiras. Por outro lado, os autores consideram que a inovação incremental proporciona às empresas um fluxo constante e seguro de vantagens provenientes de sua expertise, do "fazer o que sabemos, mas melhor".

Desta forma, argumenta-se que a profundidade da ACAP oferece vantagens consideráveis quando a empresa está interessada principalmente no refinamento de produtos existentes, buscando resultados mais confiáveis e previsíveis. Alguns estudos ainda referem que a ACAP pode aumentar a frequência de inovações incrementais, tendo como justificativa o fato de que essas inovações são geradas a partir da base de conhecimento já existente na organização (Lane 
et al., 2006; Forés \& Camisón, 2015; Ferreira \& Ferreira, 2017). Diante disso, formulam-se as seguintes proposições:

P3: O efeito da amplitude da capacidade de absorção de novos empreendimentos, será maior no desempenho em inovação radical do que no desempenho em inovação incremental.

P4: O efeito da profundidade da capacidade de absorção de novos empreendimentos, será maior no desempenho em inovação incremental do que no desempenho em inovação radical.

\section{Considerações Finais}

Espera-se que os resultados que se originem dessa proposta de investigação contribuam para a literatura em diferentes aspectos. Primeiro, compreende-se que ao serem abordados as relações dos temas aqui propostos, os resultados possibilitarão avançarmos na literatura de empreendedorismo, capacidades dinâmicas, e estratégia, possibilitando assim a compreensão de como novos empreendimentos absorvem conhecimento e sua relação com o desempenho em inovação. Em segundo lugar, a contribuição baseia-se na compreensão do papel de dois aspectos fundamentais do conhecimento - amplitude e profundidade - demonstrando empiricamente a forte conexão entre conhecimento e inovação. Reconhece-se que essa conexão já tenha sido identificada, mas pouco se sabe sobre as características do conhecimento absorvido, especialmente no caso de novos empreendimentos inseridos em ecossistemas de inovação.

Em outra perspectiva, espera-se que os resultados possam servir de auxílio aos empreendedores, que ao criarem novos negócios e ao considerarem sua inserção em ecossistemas de inovação, no sentido de contribuir para elucidar o complexo processo da dinâmica do ecossistema. Acredita-se ainda que os resultados advindos dessa proposta evidenciem a importância da compreensão dos empreendedores sobre suas configurações locais, a fim de capturar o conhecimento necessário para construir as estratégias de seus empreendimentos, indicando assim que o tempo dedicado à exploração de fontes externas de conhecimento se mostra profícuo.

Em síntese, espera-se que este estudo auxilie os diferentes atores que compõem um ecossistema de inovação no sentido de compreenderem como maximizar seus resultados de desempenho em inovação, por exemplo, ao empregarem esforços em atividades que promovam a sinergia entre os diferentes atores, e fomente a absorção do conhecimento, sendo esta considerada uma das capacidades fundamentais para o crescimento e sobrevivência em ambientes competitivos.

\section{Referências}

Adner, R. (2006). Match your innovation strategy to your innovation ecosystem. Harvard Business Review, 84(4), 98-107.

Adner, R, \& Euchner, J. (2014). Innovation Ecosystems. Research-technology Management, 6(57), 10-14. doi:10.5437/08956308X5706003. 
Adner, R., \& Kapoor, R. (2010). Value creation in innovation ecosystems: how the structure of technological interdependence affects firm performance in new technology generations. Strategic Management Journal, 31, 306-333. doi:10.1002/smj.821.

Autio, E., \& Thomas, L. (2014). Innovation ecosystems. Oxford Handbook of Innovation Management, 204-288.

Chesbrough, H. (2010). Business Model Innovation: Opportunities and Barriers. Long Range Planning, 43, 354-363. doi:10.1016/j.lrp.2009.07.010.

Cohen, W. M., \& Levinthal, D. A. (1989). Innovation and learning: the two faces of R\&D. Economic Journal, 99(397), 569-596.

Cohen, W. M., \& Levinthal, D. A. (1990). Absorptive-Capacity - A New Perspective on Learning and Innovation. Administrative Science Quarterly, 35(1), 128-152.

Escribano, A., Fosfuri, A., \& Tribó, J. A. (2009). Managing external knowledge flows: the moderating role of absorptive capacity. Research Policy, 38 (1), 96-105. doi:10.1016/j.respol.2008.10.022.

Farazi, M. S., Gopalakrishnan, S., \& Perez-Luño, A. (2019). Depth and breadth of knowledge and the governance of technology alliances. Journal of Engineering and Technology Management, 54 (2019) 28-40. doi:10.1016/j.jengtecman.2019.08.002.

Ferreira, J., Raposo, M., \& Fernandes, C. (2013). Does innovativeness of knowledge-intensive business services differ from other industries? The Service Industries Journal, 33(7-8), 734748, doi:10.1080/02642069.2013.740462.

Ferreira, J., Fernandes, C., Alves, H., \& Raposo, M. (2015). Drivers of innovation strategies: Testing the Tidd and Bessant (2009) model. Journal of Business Research, 68 (7), 1395-1403. doi.org/10.1016/j.jbusres.2015.01.021.

Ferreira, G. C., \& Ferreira, J. J. M. (2017). Absorptive capacity: An analysis in the context of brazilian family firms. RAM, Rev. Adm. Mackenzie (Mackenzie Management Review), 18(1), 174-204. doi:10.1590/1678-69712017/administracao.

Ferreira, G. C., \& Ferreira, J. J. M. (2020). Generational Diversity as a Moderator for the Relationship between Absorptive Capacity and Innovation Performance at Family Firms. In: Saiz-Álvarez, J. M.; Leitão, J.; Palma-Ruiz, J. M. (Org.). Entrepreneurship and Family Business Vitality Surviving and Flourishing in the Long Term. Ed. Springer International Publishing, 85-111. doi:10.1007/978-3-030-15526-1.

Forés, B., \& Camisón, C. (2015). Does incremental and radical innovation performance depend on different types of knowledge accumulation capabilities and organizational size? Journal of Business Research, 69(2), 831-848. doi:10.1016/j.jbusres.2015.07.006.

Fosfuri, A., \& Tribó, J. (2008). Exploring the Antecedents of Potential Absorptive Capacity and Its Impact on Innovation Performance. Omega, 36(2), 173-187. doi:10.1016/j.omega.2006.06.012.

Freeman, C. (1988). Japan: A New National System of Innovation? In: Technical Change and Economic Theory. Dosi, G.; Freeman, C.; Nelson, R.; Silverberg, G. e Soete, L. (Eds), Printer, London. 
Gawer, A., \& Cusumano, M. A. (2014). Industry platforms and ecosystem innovation. Journal of Product Innovation Management, 31(3), 417-433. doi:10.1111/jpim.12105.

George, G., Zahra, S. A., \& Wood, D. R. (2002). The effects of business-university alliances on innovative output and financial performance: a study of publicly traded biotechnology companies. J. Bus. Ventur. 17(6), 577-609. doi:10.1016/S0883-9026(01)00069-6.

Helfat, C. E., \& Lieberman, M. B. (2002). The birth of capabilities: Market entry and the importance of prehistory. Industrial and Corporate Change, 11(4), 725-760. doi:10.1093/icc/11.4.725.

Helfat, C. E., \& Winter, S. G. (2011). Untangling dynamic and operational capabilities: strategy for the (n)ever-changing world. Strategic Management Journal, 32(11), 1243-1250. doi:10.1002/smj.955.

Kor, Y. Y., \& Mesko, A. (2013). Dynamic managerial capabilities: Configuration and orchestration of top executives' capabilities and the firm's dominant logic. Strategic Management Journal, 34(2), 233-244. doi:10.1002/smj.2000.

Lane, P. J., Koka, B., \& Pathak, S. (2006). The Reification of Absorptive Capacity: a critical review and rejuvenation of the construct. Academy of Management Review, 31(4), 833-863. doi:10.5465/AMR.2006.22527456.

Larrañeta, B., Galán González, J. L., \& Aguilar, R. (2016). Early efforts to develop absorptive capacity and their performance implications: Differences among corporate and independent ventures. The Journal of Technology Transfer. doi:10.1007/s10961-016-9488-1.

McDougall, P. P., Robinson, R. J., \& DeNisi, A. S. (1992). Modelling new venture performance: An analysis of new venture strategy, industry structure, and venture origin. Journal of Business Venturing, 7(4), 267-290.

Moore, J. F. (1993). Predators and prey: A new ecology of competition. Harvard Business Review, 71(3), 75-86.

Moore, J. F. (1996). The Death of Competition: Leadership and strategy in the age of business ecosystems. New York, NY: Harper Business.

Nambisan, S., \& Baron, R. A. (2013). Entrepreneurship in Innovation Ecosystems: Entrepreneurs' Self-Regulatory Processes and Their Implications for New Venture Success. Entrepreneurship Theory and Practice, 37, 1071-1097. doi:10.1111/j.15406520.2012.00519.x.

OCDE (2005). Organização para Cooperação e Desenvolvimento Econômico. Manual de Oslo: diretrizes para coleta e interpretação de dados sobre inovação. 3.ed. Brasil: Ministério da Ciência e Tecnologia.

Patel, P. C., Criaco, G., \& Naldi, L. (2016). Geographic diversification and the survival of bornglobals. Journal of Management, 44(5), 1-29. doi:10.1177/0149206316635251.

Porter, M. (1998). Clusters and the new economics of competition. Harvard Business Review, 76(6), 77-90.

Porter, M. (2003). The economic performance of regions. Regional Studies, 37, 549-578. 
Ritala, P., \& Hurmelinna-Laukkanen, P. (2013). Incremental and Radical Innovation in Coopetition-The Role of Absorptive Capacity and Appropriability. Journal of Product Innovation Management, 30, 154-169. doi:10.1111/j.1540-5885.2012.00956.x.

Spinosa, L. M., Schlemm, M. M, \& Reis, R. S. (2015). Brazilian innovation ecosystems in perspective: some challenges for stakeholders. REBRAE, 8(3), 386-400. doi:10.7213/rebrae.v8i3.14174.g13607.

Srivastava, M. K., \& Gnyawali, D. R. (2011). When do relational resources matter? Leveraging portfolio technological resources for breakthrough innovation. Academy of Management Journal. 54 (4), 797-810. doi:10.5465/AMJ.2011.64870140.

Tidd, J., \& Bessant, J. (2015). Gestão da Inovação [F. Nonnenmacher, Trad.]. (5a ed.). Porto Alegre: Bookman.

Vasudeva, G., \& Anand, J. (2011). Unpacking absorptive capacity: A study of knowledge utilization from alliance portfolios. Academy of Management Journal, 54(3), 611-623.

Velu, C. (2015). Knowledge management capabilities of lead firms in innovation ecosystems. AMS Review, 5 (3-4), 123-141. doi:10.1007/s13162-015-0068-6.

Zahra, S. A., \& George, G. (2002). Absorptive Capacity: a review, reconceptualization, and extension. Academy of Management Review, 27(2), 185-203. doi:10.5465/AMR.2002.6587995.

Zahra, S. A. (2008). The virtuous cycle of discovery and creation of entrepreneurial opportunities. Strategic Entrepreneurship Journal, 2 (1), 243-257. doi:10.1002/sej.47.

Zahra, S. A., \& Nambisan, S. (2011). Entrepreneurship in global innovation ecosystem. Academy of marketing science review, 1(1), 4-17. doi:10.1007/s13162-011-0004-3.

Zahra, S. A., Larraneta, B., \& Galán, J. L. (2015). Absorptive Capacity and Technological Innovation. Wiley Encyclopedia of Management. 13, 1-5. doi:10.1002/9781118785317.weom130020.

Zhang, J., \& Baden-Fuller, C. (2010). The influence of technological knowledge base and organizational structure on technology collaboration. Journal Management Studies, 47 (4), 679-704. doi:10.1111/j.1467-6486.2009.00885.x.

Zhang, J., Baden-Fuller, C., \& Mangematin, V. (2007). Technological knowledge base, R\&D organization structure and alliance formation: evidence from the biopharmaceutical industry. Research Policy, 36 (4), 515-528. doi:10.1016/j.respol.2007.02.015. 